\title{
Towards Quantum Limited Frequency Multiplication
}

\author{
H. Rashid, V. Desmaris, A. Pavolotsky, V. Belitsky \\ Chalmers University of Technology, Gothenburg, Sweden
}

\begin{abstract}
This paper presents a tentative modelling of Superconductor-Insulator-Superconductor (SIS) junction used as frequency multiplier. The model is tested against the experimental data presented earlier and demonstrates good agreement between the measured data and simulation. Such a model will improve understanding of the SIS junction used as frequency multiplier and hopefully lead us to demonstration of the performance for such multiplier.
\end{abstract}

\section{INTRODUCTION}

$\mathrm{M}$ ODERN cryogenic heterodyne millimeter and -sub millimeter receivers for radio-astronomy employ Superconductor-Insulator-Superconductor (SIS) tunnel junctions as mixers. The noise contribution from the mixer is greatly reduced due to quantum nature of the SIS tunnel junction operation. The SIS mixer in the quantum mode enables it to operate with conversion gain whereas the tunneling current voltage (I-V) characteristic of the junction exhibits extremely sharp quasi-particle nonlinearity [1]. This technology provides ultimate sensitivity and possibilities for spectral line (spectroscopic) observations [2, 3].

There have been extensive studies, both theoretically and experimentally, to understand the device physics and its modeling of the SIS as mixer. However, little work has been done in modelling and understanding of SIS junction as frequency multiplier [4]. Recently, Billade et al. [5] demonstrated a frequency doubler using a series SIS array consisting of 68 elements. The experiment revealed the desired frequency multiplication; however, the overall conversion efficiency was poor and only few nano-watts of power could be delivered at the output frequency.

If we consider such array multiplier, the 68 element series SIS array becomes electrically long and must be regarded as distributed. The pumping power will, therefore, decay along the array such that any preceding element in the series SIS array is pumped harder than the next element. Furthermore, there is no reason to believe that the temperature for each element would stay unchanged in such conditions, especially for the SIS junctions situated closer to the point of injecting the pumping signal where power per junction would be the highest. Therefore, it is likely that there exists a temperature gradient along the SIS elements. The temperature deviation (increase) yields a smearing of the density of states for the superconductor electrodes of the junction, which leads to degradation of the gap voltage of the SIS junction and the gap parameter $\delta \mathrm{Vg}$. This is clearly seen in Fig. 2, which is reproduced from [5]. Here we present results of using different approach to modelling of SIS junction multiplier: instead of using time-domain pumping signal waveform fitting procedure, we use close form solutions generated harmonics from small-signal approximation. The deviations of the smallsignal model from large signal model are accounted by limiting the pumping level of the SIS junction.

\section{MODEL}

In an attempt to better model the measured data from [5], a new small-signal model was made. The small-signal model employs frequency domain equations for the induced tunnel current [1]. The purpose here is to get a quantitative insight of the measurements performed in [5], which can later be used for a more detailed large signal simulation. The pumping factor, $\alpha$, poses one of the limits in the small signal model. The small signal model should give adequate qualitative results when the pumping factor is below 3, however for the accurate quantitative simulations the differences between large- and small-signal models must be taken into account.

It is a known fact that the reactive portion of the tunneling current and induced the current can be obtained with just the knowledge of the DC quasi-particle tunnel current characteristics, i.e., the DC IVC. The reactive part of the tunneling current is obtained with the Kramers-Kronig transform of the DC IVC [1]. In order to ease simulations, we developed a novel model using a similar approach as in [6] (but with much fewer parameters) for generating the DC IVC, instead of using measured DC IVC. By varying the parameters in this model, we are able to study and understand the SIS multiplication (also mixing) process in much more detail.

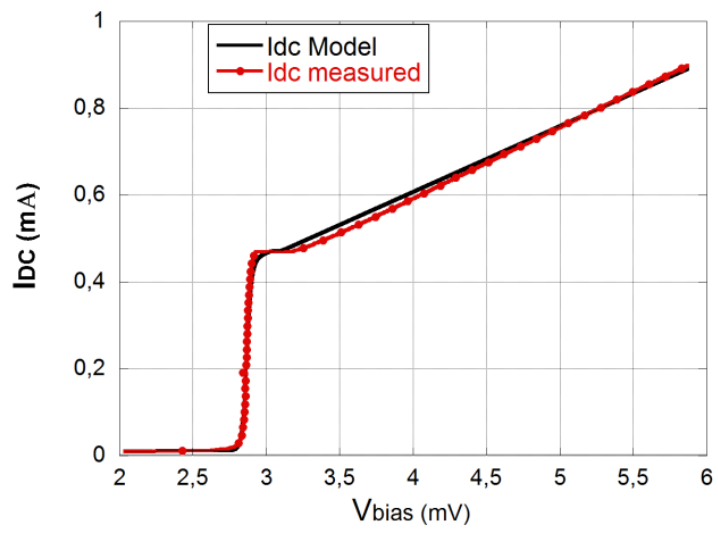

Fig.1: Modelled and measured IVC.

Different multiplication factors of the SIS junction based multiplier were studied by implementing the DC IVC model in to the small signal model. However, we have thus far, mainly studied the second harmonic in order to compare with measurements performed in [5] and tune the new model for better accuracy. 
The simulations of the frequency doubler (single junction) made with our model show the characteristic behavior of the multiplier, which is also present in the measured multiplier [5]. The experimental data with 68 SIS junction series array were scaled to single junction at the voltage scale.

Fig. 3 shows the estimated and measured output power of the distributed SIS array [5]. In Fig.4 we can clearly see one large peak close to $2.5 \mathrm{mV}$ and two smaller peaks near $3.1 \mathrm{mV}$ and $3.5 \mathrm{mV}$, which is consistent with the measured performance shown in Fig.3. The corresponding peaks in Fig.3 are found approximately at $2.7 \mathrm{mV}, 2.9 \mathrm{mV}$ and $3.5 \mathrm{mV}$. The small signal model also predicts the sharp dip at $2.87 \mathrm{mV}$.

At this stage, the new small signal model does not provide a perfect fit to the measurements data. On the other hand, our model here treats only one isolated SIS junction whereas experimental data are related to the distributed SIS array, where each junction is exposed to different pumping signal power and consequently has different physical temperatures, the pumping signal and its product, the second harmonic has different phase at each junction of the series array. In order to get better fit of the model, we included temperature effects for different junctions, and we were able to also explain the broadening of the first peak near $2.5 \mathrm{mV}$ in Fig.3.

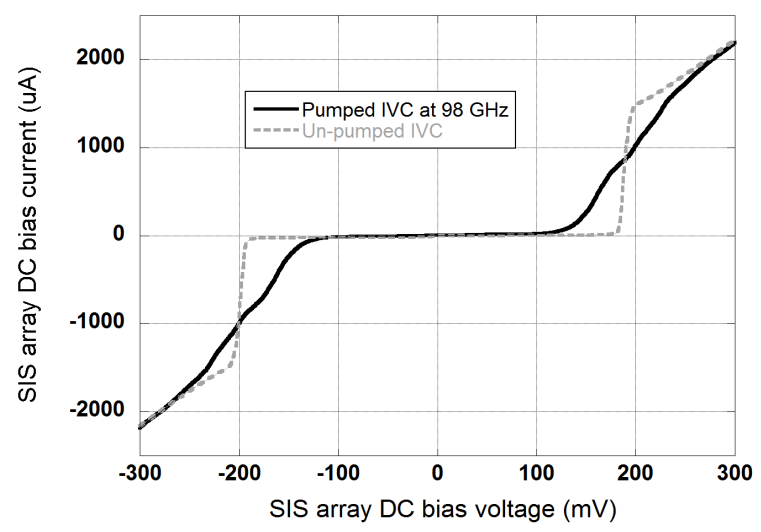

Fig.2: overheating of the SIS array at a high level of applied pumping signal. This figure is reproduced from [5].

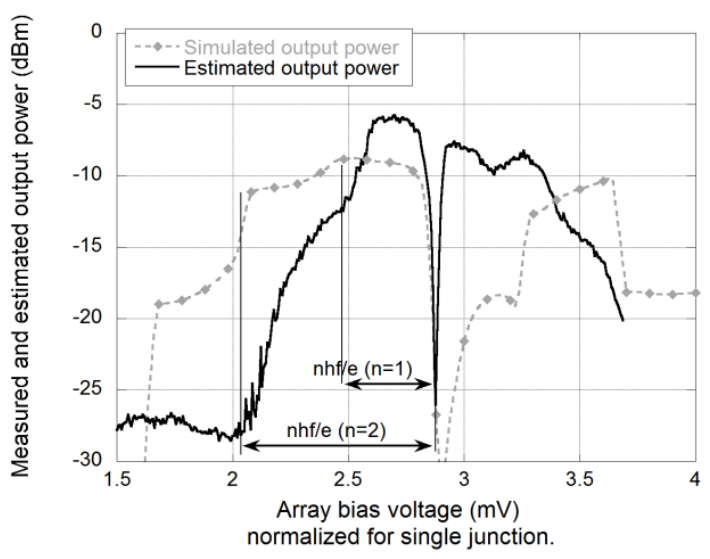

Fig.3: Bias voltage dependence of the multiplied signal. With 190-GHz LO frequency for the SIS mixer and while sweeping the SIS array bias voltage, the power of the 196-GHz signal was measured (solid black curve) at 6-GHz IF using a spectrum analyzer. The voltage axis for the entire array is normalized for single junction. The dashed gray curve shows the estimated output power from the model. The figure is reproduced from [5].

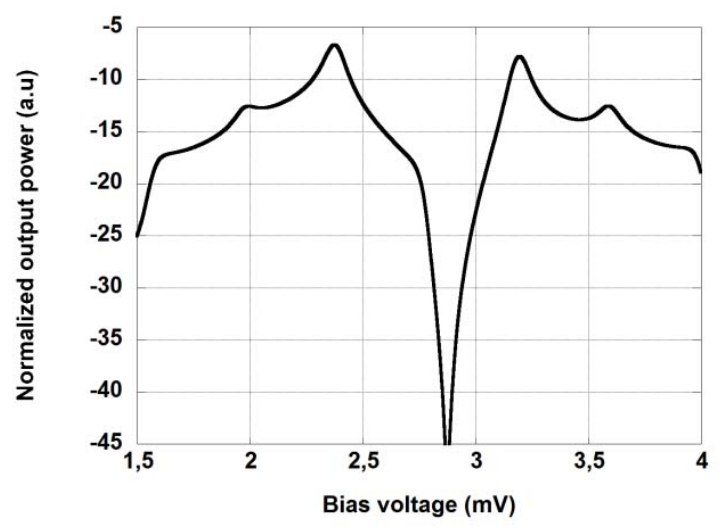

Fig.4: The normalized output power for a single junction from our smallsignal model.

Figure 4 shows the estimated output power (normalized) from our small-signal model, whereas the black and gray curves in Fig. 3 shows the reproduced measured and estimated output power from [5].

\section{CONCLUSION}

We have created a frequency domain small signal model based on Tuckers mixer theory. We have also developed a novel model for generating the DC IVC, instead of using measured DC IVC, which enables us to study and understand the SIS multiplication process in much more detail by studying the dependence of the multiplication vs. model parameters. The characteristic features of the estimated output power of a single SIS junction from our model are very similar to experimental data previously reported.

\section{ACKNOWLEDGEMENT}

Authors would like to thank Dr. Bhushan Billade for sharing measurement data of the SIS multiplier and useful discussions.

\section{REFERENCES}

J. R. Tucker, "Quantum limited detection in tunnel junction mixers," Quantum Electronics, IEEE Journal of, vol. 15, pp. 12341258, 1979.

V. Belitsky, I. Lapkin, V. Vassilev, R. Monje, A. Pavolotsky, D. Meledin, et al., "Facility heterodyne receiver for the Atacama Pathfinder Experiment Telescope," in Infrared and Millimeter Waves, 2007 and the 2007 15th International Conference on Terahertz Electronics. IRMMW-THz. Joint 32nd International Conference on, 2007, pp. 326-328.

B. Billade, O. Nystrom, D. Meledin, E. Sundin, I. Lapkin, M. Fredrixon, et al., "Performance of the First ALMA Band 5 Production Cartridge," Terahertz Science and Technology, IEEE Transactions on, vol. 2, pp. 208-214, 2012.

H. D. Foltz and J. H. Davis, "Calculated performance of SIS junctions as frequency multipliers," in Microwave Symposium Digest, 1990., IEEE MTT-S International, 1990, pp. 307-310 vol.1. B. Billade, A. Pavolotsky, and V. Belitsky, "Experimental Study of Frequency Multiplication in a Distributed Array of SIS Junctions," Terahertz Science and Technology, IEEE Transactions on, vol. 4, pp. 254-259, 2014.

A. B. Ermakov, V. P. Koshelets, I. L. Serpuchenko, L. V. Filippenko, S. V. Shitov, and A. N. Vystavkin, "SNAP structures with $\mathrm{Nb}-\mathrm{AlO}<$ sub $>\mathrm{x}</$ sub $>-\mathrm{Nb}$ junctions for MM-wave receivers," Magnetics, IEEE Transactions on, vol. 25, pp. 1060-1063, 1989. 